\title{
Deterministic Transfection Drives Efficient Nonviral Reprogramming and Uncovers Reprogramming Barriers
}

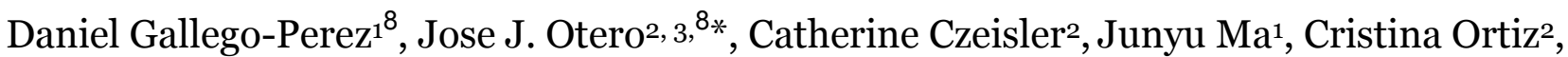
Patrick Gygli², Fay Patsy Catacutan², Hamza Numan Gokozan², Aaron Cowgill², Thomas Sherwood3, Subhadip Ghatak4, Veysi Malkoc'1, Xi Zhao¹, Wei-Ching Liao¹, Surya Gnyawali4, Xinmei Wang1, Andrew F. Adler5,6, Kam Leong5,7, Brian Wulff², Traci A. Wilgus², Candice Askwith33, Savita Khanna4, Cameron Rink4, Chandan K. Sen4*, L. James. Lee ${ }^{*}$

\footnotetext{
Affiliations:

${ }^{1}$ The Ohio State University, College of Engineering, Department of Chemical and Biomolecular Engineering, Columbus, OH.

${ }^{2}$ The Ohio State University, College of Medicine, Department of Pathology, Columbus, OH.

3The Ohio State University, College of Medicine, Department of Neuroscience, Columbus, $\mathrm{OH}$.

4The Ohio State University, College of Medicine, Department of Surgery, Columbus, OH.

5Duke University, Department of Biomedical Engineering, Durham, NC.

${ }^{6}$ Current address: Department of Neurosciences, University of California - San Diego, La Jolla, CA, United States of America, 92093

7Current address: Department of Biomedical Engineering, Columbia University, New York, NY
}

\section{Equal contribution}

*Authors of Correspondence

\begin{tabular}{|l|l|l|}
\hline L. James Lee, Ph.D. & Chandan K. Sen, Ph.D. & José Javier Otero, M.D., Ph.D. \\
488 Koffolt Laboratories & 473 W 12th Ave & Associate Director of \\
CBEC & Columbus, OH 43210 & Neuropathology \\
151 W Woodruff Ave & sen.16@osu.edu & 4169 Graves Hall \\
Columbus, OH 43210 & & 333 W 10 \\
Tel: 614-292-2408 & & Columbus, OH 43210 \\
lee.31@osu.edu & & Tel: 614-685-6799 \\
& & Fax: 614-292-5849 \\
& & jose.otero@osumc.edu \\
\hline
\end{tabular}

Editorial Communication from Cell Reports is preferred with José Javier Otero, M.D., Ph.D.

Format: Original Article Submission to Nenomedicine: Nanotechnology, Biology, and Medicine.

Figure Count: 6

Table Count: o

Abstract word count: 150 .

Manuscript word count (background, experimental procedures, results, discussion): 4996.

Number of references: 27

\section{Acknowledgements}

Funding provided by NIBIB (1R21EBo17539-01A1 and 3R21EB017539-01A1S1), National

Science Foundation (NSEC EEC-0914790), National Center for the Advancing Translational Sciences (UL1TRoo1070 and 8UL1TRoooo90-05), NINDS P30 core grant (NS045758) and NIH GM069589, GM077185, GM108014, NS42617 and NR 013898. The content is solely the responsibility of the authors and does not necessarily represent the official views of the National Center for Advancing Translational Sciences, National Science Foundation or the National Institutes of Health. This work was sponsored by and represents activity of The Ohio State University Nanoscale Engineering Center for Affordable Nanoengineering of Polymeric Biomedical Devices and Center for Regenerative Medicine and Cell Based Therapies (regenerativemedicine.osu.edu). 


\begin{abstract}
Safety concerns and/or the stochastic nature of current transduction approaches have hampered clinical translation of nuclear reprogramming. We report a novel non-viral nanotechnology-based platform permitting deterministic large-scale transfection with single-cell resolution. The superior capabilities of our technology are demonstrated by modification of the well-established direct neuronal reprogramming paradigm using overexpression of the transcription factors Brn2, Ascl1, and Myt1l (BAM). Reprogramming efficiencies were comparable to viral methodologies (up to $\sim 9-12 \%$ ) without the constraints of capsid size and with the ability to control plasmid dosage, in addition to showing superior performance relative to existing non-viral methods. Furthermore, increased neuronal complexity could be tailored by varying BAM ratio and by including additional proneural genes to the BAM cocktail. Furthermore, high-throughput NEP allowed easy interrogation of the reprogramming process. We discovered that BAM-mediated reprogramming is regulated by AsclI dosage, the S-phase cyclin $C C N A 2$, and that some induced neurons passed through a nestin-positive cell stage.
\end{abstract}




\section{Background}

Directly inducing a specific cell fate requires not only the knowledge of which factors can stably induce a desired cell type, but also the ability to introduce these exogenous genes/proteins into cells in a controlled fashion. As has been elegantly demonstrated, nuclear reprogramming methodologies typically require relatively small cocktails of transcription factors to change a cell's epigenetic framework. For example, exogenous expression of OCT4, KLF4, SOX2, and cMYC reprograms fibroblasts into induced pluripotent stem (iPS) cells ${ }^{1}$. In addition, direct nuclear reprogramming of fibroblasts into post-mitotic neurons has been demonstrated by the forced expression of Ascl1, Brn2, and Myt1 ${ }^{2}$. These technologies provide significant opportunities to improve human health by advancing our understandings of basic cell biology, by facilitating the modeling of human diseases for pre-clinical drug development pipelines, and, ultimately, for clinically-relevant cell therapies.

For regenerative medicine and disease modeling applications, it may be more desirable to induce cells directly to the lineage of interest rather than by first proceeding through an iPS cell stage. This represents a unique challenge in cases where the cell of interest is post-mitotic and underscores the need to discover deterministic transfection methodologies for reprogramming applications. For example, to obtain a high quantity of neurons from direct neuronal reprogramming of dermal fibroblasts, a large number of cells would have to undergo the reprogramming process deterministically. Such reprogramming strategies would require the ability to control the transdifferentiation of large numbers of cells. Although significant advances have been made in reprogramming paradigms, including the implementation of small molecules and pharmaceutical approaches $3{ }^{4-7}$, the ability to directly control the dosage of reprogramming factors in each individual cell has not yet been achieved. Furthermore, the stochastic barriers to implementing this long term vision have not been identified. 
The most widely implemented non-viral transfection approaches depend upon diffusion- and endocytosis-based mechanisms, resulting in stochastic transfections ${ }^{8}$. We have overcome this problem by developing a novel and easy-to-use device that permits in situ electroporation on cultured cells through a 3D ordered array of nanochannels. Such an array was created via the adaptation of conventional transwell inserts, which are commonly used in routine cell culture, for nanochannel electroporation (NEP). NEP is a single-cell transfection system that electrophoretically delivers charged agents directly into the cytosol by applying an intense electric field over a small area of the cell membrane 9 , thus facilitating precise dosage control with minimum cell damage. A significant advantage of NEP over other electroporation paradigms is the benign nature of the transfection, resulting in negligible perturbations to cell physiology9. Although other electroporation modalities have similarly demonstrated enhanced transfection control of various types of engineered molecules with minimal cell toxicity ${ }^{10,11}$, deterministic transfection technologies for nuclear reprogramming applications has remained elusive. We validated the superiority of our novel NEP platform with a well-established direct nuclear reprogramming model that involves fibroblast transdifferentiation into neurons by exogenous expression of Brn2, AsclI, and Myt1l (BAM). We found that the NEP platform was superior to BEP with regards to reprogramming efficiency, showing iN generation at levels comparable to reports using lentivirus. Moreover, due to the inherent deterministic advantages of the NEP methodology, we were also able to demonstrate that nuclear reprogramming by BAM is regulated by AsclI dosage, is regulated by the S-phase cyclin CCNA2, can be enhanced by adding proneural genes to the NEP cocktail, and that at least some neurons induced through BAM-mediated neuronal reprogramming may pass through a nestin-positive cell stage. 


\section{Methods}

\section{NEP Platform Fabrication:}

Unmodified transwell inserts possess a highly dense and random array of nanochannels whose interactions with plated cells cannot be controlled at the single cell level. This could potentially result in high intercellular variability in the transfection characteristics among other things. Using standard cleanroom technologies (Fig. 1, Fig. S1), the apical or basal surface of the Transwells were spin coated (CE 10oCB Resist Coater) with positive photoresist (S1813 or SPR-220.7) at $~ 3000 \mathrm{rpm}$ for $1 \mathrm{~min}$. The devices were subsequently soft-baked at room temperature for $>16 \mathrm{~h}$. The resist was then controllably exposed to UV light through a dark field photomask with circular openings of varying diameters using a contact aligner (EV Group 620 Advanced Contact Aligner). Following exposure, the photoresist was developed in positive resist developer for 1-2 min, which exposed the underlying nanopores on the Transwell membrane in an arrayed and orderly manner. Further details of the fabrication procedure are presented in the supplemental materials.

\section{Cell Culture and Transfections:}

BEP-based transfection: A Neon (Life Technologies) transfection system was used for BEP transfection. The cells were resuspended at a final concentration of $1.0 \times 10^{\wedge} 6$ cells, in $0.1 \mathrm{ml}$. The cell suspension was then mixed with the plasmid (0.05-0.1 $\mu \mathrm{g} / \mu \mathrm{l})$ solution, and the tip was then inserted into the Neon tube containing the electrolytic buffer. The cells were electroporated following a protocol suggested by the manufacturer (one $30 \mathrm{~ms}$ pulse of $1350 \mathrm{~V}$ ) optimized for MEF cells. BEP-based transfection outside these conditions could result in reduced efficacy and/or increased MEF cell death.

NEP-based transfection: MEF cells were directly loaded on the entire apical surface of the Transwell membrane at a density of $\sim 1.0-1.5 \times 10^{4}$ cells $/ \mathrm{cm}^{2}$ in regular maintenance medium (Fig. 1A). The cells were allowed to adhere and spread overnight before NEP transfection. 
Alternatively, cells could be selectively plated directly on top of the exposed nanochannels on the apical surface of the Transwell by controllably applying dielectrophoretic forces across the Transwell (Fig. S1). Following cell loading, the media in the apical chamber was replaced by PBS and the Transwell inserts were then mounted on an e-beam evaporated (Denton DV-502A E-Gun Evaporator) gold electrode that was in direct contact with the plasmid solution (Fig. 1C). A counter-electrode was then immersed in the PBS of the apical chamber, and a square wave pulse (250 V, $20 \mathrm{~ms}$ duration pulse, 1-10 pulses) was applied across the electrodes using a Biorad Gene Pulser Xcell power supply. The PBS was replaced by fresh media immediately after, and the cells were then incubated overnight at $37 \mathrm{C}$.

Induced Neuron protocol: Post-NEP, MEF's were cultured in N3 media. N3 neuronal induction medium was prepared by supplementing DMEM/F-12 (Invitrogen) base media with $25 \mu \mathrm{g} / \mathrm{ml}$ bovine insulin, $30 \mathrm{nmol} / \mathrm{l}$ sodium selenite, $50 \mu \mathrm{g} / \mathrm{ml}$ human apo-transferrin, 20 $\mathrm{nmol} / \mathrm{l}$ progesterone, $10 \mathrm{ng} / \mathrm{ml}$ human $\mathrm{bFGF} 2$, and $100 \mu \mathrm{mol} / \mathrm{l}$ putrescine.

\section{Statistical Analysis}

All statistics were performed in Rv2.12, Microsoft Excel, and Matlab. Sholl analysis and dendrite complexity index were performed using Neurolucida Explorer ${ }^{\mathrm{TM}} \mathrm{V}_{\mathrm{V} 11}$. 


\section{Results}

\section{An easy-to-use platform for deterministic large scale cell transfection}

Fig. 1A illustrates the device design. Briefly, an array of nano- to micro-scale wells were patterned on the nanochanneled transwell membrane surface to ( $i$ ) minimize cytotoxic currents during electrotransfection, and (ii) to better control the extent of interaction of single cells with the nanoporating channels (Fig. S1A). This in turn leads to controlled dosage at the single cell level. The cells are then loaded on the apical surface, which is subsequently mounted within a pair of electrodes over a reservoir containing the cargo solution (Fig. 1C). A square wave DC pulse with a $250 \mathrm{~V}$ amplitude and a $20 \mathrm{~ms}$ pulse duration is then applied across the electrodes to porate the cells (Fig. 1D-G) directly delivering cargo into the cytosol.

This 3D NEP platform was validated by transfecting three cDNA expression constructs at a 1:1:1 molar ratio as model cargo into mouse embryonic fibroblasts (MEF). We utilized reprogramming constructs designed with bicistronic fluorescent reporters; signals in cyan (Myt1l), red (Brn2), and green (Ascl1) indicated successful transcription of BAM mRNA. Bulk electroporation (BEP) ${ }^{12}$ was used as a control transfection method. Although BAM transfection by NEP or BEP resulted in relatively early $(\sim 4 \mathrm{~h})$ Ascl1 plasmid expression, NEP-based transfection led to significantly higher and more uniform transfection extent compared to BEP (Fig. S1P). Moreover, NEP conditions could be easily tailored to control the dosage (Fig. S1P3). We compared NEP-treated cells with the upper 2\% BEP-transfected cells (as sorted by FACS of GFP-positive cells) and found that even though the mean RFU of this BEP subset was similar to that of the NEP 5-pulse group, the NEP-treated cells had improved viability and a smaller interquartile range in RFU relative to the BEP cells (Fig. S1Q). The top 2\% BEP cells were viable for less than 2 days.

Co-expression of all three plasmids was detected $24 \mathrm{~h}$ after transfection. When comparing GFP/RFP/CFP-positive cells, NEP 5-pulse resulted in significantly higher mean relative 
fluorescence expression compared to BEP (Fig. 1H-J). Moreover, the fluorescence intensity variance among NEP-treated cells was significantly lower compared to BEP. In addition, while all the clones in the NEP group had a tendency to successfully co-express all three plasmids, a host of cells in the BEP group only showed detectable expression of one or two of the three plasmids (Fig. 1 H1-J1 arrowheads). Persistent long-term expression of BAM reporter constructs was seen in a number of cells in the NEP group (Fig. 2). This was further confirmed by in vivo studies in which MEFs underwent NEP of $B A M$ prior to being subcutaneously injected into E15.5 embryos in utero. Postnatal day 4 pups that had undergone embryonic transplanation showed significant GFP expression by in vivo IVIS imaging (Fig. 2) compared to control pups. We conclude that NEP-based transfection results in stronger and more uniform gene expression compared to BEP, and that such expression showed stable and long-lasting behavior both in vitro and in vivo.

\section{Deterministic NEP-based transfection leads to efficient and controlled}

\section{transfection}

In order to further test the effects of a more deterministic transfection approach in reprogramming outcome, we cultured the transfected MEFs in N3 media for a prolonged period of time and monitored induced neuron (iN) formation by assaying neuronal marker expression, morphological changes, and electrophysiological activity. NEP-treated cultures showed significantly higher and more narrowly-distributed early (day 7) reprogramming efficiencies (based on number of TUJ1+ cells) across samples compared to BEP (Fig. 3A). Following transfection, a number of NEP-treated cells started to show significant morphological changes compatible with neuronal transformation within remarkably short timeframes ( $<48 \mathrm{~h}$, Supplementary Video 1), exhibiting slightly faster kinetics compared to viral infection ${ }^{2}$. NEP of human dermal fibroblasts with BAM plasmids also showed rapid morphological changes suggestive of reprogramming (Fig. S1R). Mature neuronal marker (MAP2, synapsin and 
neurofilament) expression in the NEP group was similarly detected very early post-NEP (week 1). Moreover, NEP-treated cells also showed electrophysiological activity as demonstrated by their capacity to undergo induced action potentials (Fig. 3E-H).

A single NEP-based transfection was enough to reprogram a higher proportion of iNs in a shorter period of time compared to previous reports on non-viral iN reprogramming ${ }^{13}$. Nevertheless, a relatively large proportion of MEFs still had not shown signs of iN reprogramming at our experimental end-points. We hypothesized that this was partly due to the fact that Ascl1 plasmid expression appears to be rapidly and selectively silenced in a number of MEF cells (Supplementary Video 2). Brn2 and Myt1l expression on the other hand seemed to be less prone such silencing.

We therefore increased the dosage of Ascl1 relative to Brn2 and Myt1l in NEP so as to determine a more optimal dosage of $B A M$ cDNA expression plasmids for neuronal reprogramming. The current status quo for controlling molar ratios of reprogramming genes is to deliver them in a multicistronic expression plasmid ${ }^{12}$ or implement TEVP-mediated proteolytic cleavage ${ }^{14}$. We found that increasing the relative molar ratio of Ascl1 to 2:1:1 (Ascl1:Brn2:Myt1L) resulted in a more sharply enhanced reprogramming efficiency in the NEP group compared to BEP by day 7 post-transfection (Fig. 3A). Moreover, altering the molar ratio of BAM also led to increased neuronal morphological complexity (Fig. 3I). BEP-treated MEFs, instead, showed no clear change in morphology in response to the altered BAM ratio. We conclude that the NEP technology is a better-suited approach not only for enhanced but also for more controlled transfection and enhanced reprogramming. We also note that current technologies, including BEP and viral-based technologies, cannot achieve such tailorable reprogramming.

Prior studies have shown that specific neuronal traits could be induced by including additional proneural genes in the reprogramming cocktail ${ }^{15}$. Efficient multi-factor delivery, 
however, is extremely challenging with viral or non-viral transfection technologies. NEP on the other hand has the ability to deliver such complex combinations in a more deterministic manner at the single cell level. The unique control that NEP provides when transfecting multiple plasmids allows the rapid and well-controlled screening of many transcription factor combinations for desired phenotypes following direct reprogramming. As our model, we used information from the mammalian embryonic hindbrain development, which has a wellcharacterized gradient of dorsal-ventral and rostro-caudal patterning genes. We identified and selected additional proneural transcription factors, $P H O X 2 B, P H O X 2 A$, and $R U N X_{3}$ that are involved in patterning of autonomic hindbrain neurons 16,17 . Plasmids encoding for such factors, in combination with BAM, were then NEP-transfected into MEFs. Transfected cultures were then analyzed for marker expression and morphometry. Our results showed a slight increase in reprogramming efficiency by day 14 after transfection for the BAM/PHOX2B/PHOX2A/RUNX 3 group relative to BAM only (mean iN reprogramming efficiency $=12 \%$ for BAM-PPR and $9 \%$ for BAM alone, Fig. S2). As such, our approach achieved reprogramming efficiencies comparable to viral methodologies but without the constraints of capsid size, and with the ability to control plasmid dosage at the single cell level. We therefore analyzed neuronal maturity by morphometric analyses. Morphometric analyses of the iNs showed the most marked changes between groups, with iNs from the six-factor cocktail exhibiting more complex and mature neuronal morphologies compared to BAM alone. We conclude that NEP can improve the quality of neuronal reprogramming by increasing the morphological complexity of the neurons generated. Furthermore, since adding additional neuronal proneural genes resulted in only a modest, albeit significant, increase in neuronal reprogramming efficiency, we conclude that several barriers exist to the efficient generation of induced neurons that are not solved by more deterministic plasmid delivery.

NEP helps probe and uncover novel stochastic barriers to iN formation 
Although NEP-based transfection ultimately resulted in relatively high reprogramming efficiencies, comparable to viral methodologies, a considerable proportion of MEFs appear to be less amenable to reprogramming. We thus designed experiments to determine cellular mechanistic barriers to direct neuronal reprogramming. Our time-lapse monitoring of BAM/NEP-treated MEFs is illustrated in Fig. 4 and in videos 1-4 in the Supplemental Material. Instances of cells showing neuronal morphology could be identified as early as 15 hours postNEP (Fig. 4A). We note that some cells showed rapid and selective plasmid silencing (Supplementary Video 2 and Fig 4), and that some cells also underwent divisions coupled with asymmetric plasmid inheritance after a division (Fig. 4). We thus proceeded to use NEP to further probe the stochastic aspects of iN reprogramming by focusing on the cell cycle, and the potential role of alternative intermediary pathways in the reprogramming process.

\section{Regulatory factors upstream of Ascl1 discovered by NEP}

Ascl1 pioneer activity is in part mediated by the interaction (direct or indirect) with the trivalent chromatin signature that includes $\mathrm{H}_{3} \mathrm{~K} 4 \mathrm{me} 1, \mathrm{H}_{3} \mathrm{~K} 27 \mathrm{ac}$, and $\mathrm{H}_{3} \mathrm{~K} 9 \mathrm{me}_{3}{ }^{18}$. Therefore, although the Ascl1 DNA consensus site clearly plays a key role, Ascl1 pioneer activity would require additional factors capable of sensing certain chromatin states. We therefore utilized the NEP platform to explore the potential roles of the S-phase cyclin CCNA2 in neuronal reprogramming. CCNA2 has a well-established, albeit somewhat unappreciated role, in chromatin regulation. First, in S-phase, the chromatin landscape undergoes dramatic alterations as the entire genome is duplicated. CCNA2's role as an initiator of replication origin 19 thus insinuates a role as a chromatin sensor. With this in mind, we isolated MEFs from pups harboring a homozygous targeted $C C N A 2$ allelle that undergoes ablation after cre-mediated recombination. We used two methods to ablate the CCNA2 locus. CCNA2fl/fl MEFs were either infected with an adenovirus-cre (or adenovirus-Lacz as a negative control) after BAM NEP resulting in cre expression several hours after BAM expression (Fig. 5), or directly co- 
transfected with a pCag-cre plasmid resulting in simultaneous cre expression in the presence of BAM. The cells were kept in culture for 14 days and the extent of reprogramming was measured in terms of $\beta$-tubulin III expression (Fig. 5A-D). Although we found that the adenovirus-creinfected cultures still showed clear signs of $\beta$-tubulin III expression, the cultures that were cotransfected with the pCag-cre-GFP plasmid concurrently with BAM during NEP clearly showed impaired reprogramming efficiency, with cre-positive $C C N A 2^{f l f l}$ cells often showing weak and very diffused TUJ1 staining patterns, and persistent fibroblast-like morphology after 14 days. Moreover, RT-PCR analysis showed clear downregulation of $\beta$-tubulin III gene expression in cre-positive cultures. The implications of this are two-fold: (1) direct cytosolic injection of plasmids by NEP facilitates rapid and more synchronous co-expression of the genes of interest $(\mathrm{BAM}+\mathrm{cre})$, thus presumably resulting in CCNA2 ablation at the early stages of cell reprogramming; (2) the underlying methodology of viral transfection, on the other hand, promoted delayed cre gene expression relative to BAM likely due to both timing of infection in addition to gene delivery, resulting in subsequent late $C C N A 2$ ablation along the reprogramming pathway. Altogether these observations suggest that $C C N A 2$ could potentially be playing a more prominent role in the regulation of early events in BAM-mediated neuronal reprogramming, and that beyond a certain point along the reprogramming trajectory, CCNA2 deletion does not appear to have a marked effect on the reprogramming outcome. This dependency on CCNA2 activity raises the point that cells may have to be in the appropriate cell cycle phase when receiving exogenous reprogramming factors to successfully undergo iN induction.

\section{NEP discovers alternative intermediary pathways in the reprogramming process}

Our findings of an S-phase cyclin as a potential pathway component of BAM-mediated reprogramming raise the possibility that at least a subset of reprogramming cells undergo Sphase. As this is improbable for mature neurons, we postulated that during the transition some somatic cells may first revert to a neural progenitor phase, followed by cell amplification and/or 
differentiation. A limited number of studies with conflicting results have looked into the potential role intermediate progenitor-like stages (i.e., neural progenitor cells) could play in BAM-mediated iN reprogramming ${ }^{20}$. To further probe this, we evaluated mRNA expression of the neural stem cell marker nestin in MEFs $24 \mathrm{~h}$ after NEP-based delivery of either the Ascl1 plasmid alone, or co-transfected with Brn2 and Myt1l. We found that Ascl1 plasmid transfection dosage correlated with nestin mRNA levels (Fig. 6). Moreover, dose-controlled delivery of BAM by NEP showed a significant increase in nestin expression, which positively correlated with exogenous Ascl1 delivery/expression (Fig. 6B). In addition, immunostaining results 14 days after NEP-based delivery of BAM showed a number of nestin-positive cells that were still actively expressing exogenous Ascl1/GFP (Fig. 6A).

In order to further determine if a subset of the neurons resulting from NEP/BAM-mediated reprogramming underwent a transient neural stem cell phase, we generated MEFs carrying the

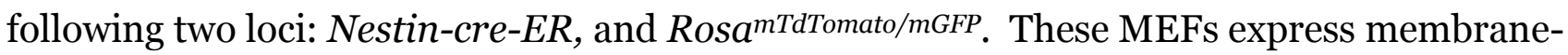
bound TdTomato in the absence of cre, whereas cells expressing a membrane-bound GFP indicate that cre-mediated excision occurred at some point in their ancestry. MEFs were treated with $1 \mu \mathrm{M}$ 4-OH to stimulate the CRE-ER construct and cells underwent BAM-mediated reprogramming by NEP. In this case we used BAM plasmids that did not carry a fluorescent reporter gene. $4-\mathrm{OH}$ was maintained in the culture media throughout the reprogramming process. Cells at 1, 7 and 14 days were fixed in 4\% paraformaldehyde, and neuronal reprogramming of GFP-positive cells was determined. These conditions showed a significant increase in the number of GFP-positive cells (Fig. 6C-E). We found many GFP-positive neurons, indicating that the cells had passed through a nestin-positive stage (Fig. 6F). We conclude that at least a subset of fibroblasts transition through a nestin-positive state during BAM-mediated neuronal reprogramming. The implications of nestin gene expression during reprograming raises the possibility that during iN generation, cells may pass through a neural 
progenitor phase. If this were to be the case, such transitions to a progenitor state would represent an additional stochastic barrier to iN generation as nestin-positive progenitor cells generate both neurons and glia. 


\section{Discussion}

\section{NEP is an Improved Methodology for Nuclear Reprogramming Applications}

The current status quo for exogenous gene expression in nuclear reprogramming all include stochastic gene expression methodologies, such as viral infection and bulk electroporation (BEP) (reviewed by Bernal ${ }^{21}$ ). In this report we have moved against the status quo by adapting nanochannel electroporation (NEP) for nuclear reprogramming. NEP's advantages include (1) high transfection delivery with low toxicity, (2) more uniform gene delivery across a group of cells, (3) transfection scalability, and (4) the capacity to transfect many reprogramming/patterning genes simultaneously. Using this approach, we discovered a novel role for CCNA2 in BAM-mediated neuronal reprogramming and have clarified the fate of MEFs during transition into neurons. Specifically, our data indicate that at least a subset of MEFs transition through a nestin-positive stage during BAM-mediated neuronal reprogramming.

Although the inclusion of multicistronic expression vectors or translated products capable of TEVP-mediated proteolysis theoretically can aid in controlling the ratios of reprogramming factors, they are fraught with caveats. First, the molar ratios achieved by implementing these methodologies will always be equimolar. Our data demonstrating that increased AsclI relative to Brn2 and MytlI increases neuronal nuclear reprogramming efficiency and increases neuronal complexity underscore the importance of controlling relative levels of reprogramming genes during this process. Furthermore, NEP technology is not constrained to plasmid DNA delivery. Indeed, any charged molecule, including drugs, miRNA ${ }^{22}$, or RNA implicated in reprogramming ${ }^{23}$, can be delivered using NEP. Although viral based gene delivery methodologies such as Sendai virus may have unique advantages, viral gene delivery will always be constrained by capsid size; such constraints are not applicable to NEP. Plasmid size 
constraints hinder efficiency of gene editing tools such as dCas9, which are often over $9 \mathrm{~kb}$ in size 24 .

\section{Stochastic Barriers to Efficient Neuronal Nuclear Reprograming}

Somatic cell reprogramming into iPS cells has some technical advantages over neuronal reprogramming. Specifically, the fact that iPS cells have a significant proliferative potential indicates that they are amenable to clonal dilution and colony picking. In contrast, somatic cell reprogramming into neurons will ultimately require a high throughput methodology to generate neurons in sufficient quantities for regenerative medicine applications. With NEP technology, we are uniquely capable of delivering in a benign fashion a precise dosage of reprogramming factors. Our findings show that NEP is an improved electroporation strategy relative to BEP in that we are capable of producing more neurons, NEP induced neurons display more complexly arborized neurites relative to bulk electroporation, and the reprogramming occurs faster. Nevertheless, with NEP we found that not all of the cells reprogrammed into neurons, and that induced neuron formation occurred at different rates, despite receiving more uniform quantities of plasmid DNA compared to BEP. Our data show that exogenous Ascl1 transcripts may be endogenously silenced more rapidly than other factors, and that some cells undergo neuronal reprogramming more rapidly than others. Furthermore, the dependency of S-phase cyclins for reprogramming research underscore a potential prominent role of the cell cycle regulatory genes. Thus, NEP BAM-mediated neuronal reprogramming has several stochastic barriers downstream of the plasmid delivery that must be overcome. Indeed, these data are in keeping with the stochastic nature of OSKM-mediated somatic cell reprogramming into iPS cells 25.

Ascl1 has been recently identified as the pioneer factor for neuronal reprogramming ${ }^{18}$.

We have shown, for the first time, that uniform expression of BAM reprogramming factors alone does not guarantee neuronal reprogramming from MEFs. Our finding showing selective depletion of exogenous Ascl1 transcription in some cells is in keeping with the notion that robust 
Ascl1 expression is required for neuronal reprogramming. Indeed, endogenous silencing of exogenous Ascl1 transcription seems to play a role in maintaining the stochastic nature to BAMmediated neuronal reprogramming. Unraveling the mechanisms of this endogenous barrier may allow the acceleration of synchronous and deterministic neuronal reprogramming as has been shown in iPS cell reprogramming paradigms ${ }^{26}$. Nevertheless, the cellular mechanisms by which Ascl1 promotes neuronal reprogramming are unknown. In the chick developing neural tube, exogenous murine Ascl1 expression accelerates neuronal differentiation with concomitant cell cycle exit ${ }^{27}$. In contrast, in the murine ventral telencephalon, several pro-proliferative genes are enhanced directly by Ascl1, including several G1/S and G2/M-transition proteins; these genes are activated prior to classical Ascl1-associated genes implicated in neuronal specification with cell cycle exit ${ }^{28}$. It is therefore not surprising that targeted ablation of the S-phase gene CCNA2 early during neuronal reprogramming hampers iN formation. These data suggest that early during the process, at least some reprogramming cells may require DNA replication, which is a known modifier of epigenetic signatures ${ }^{29}$. Furthermore, our data suggest that at least a subset of reprogrammed neurons proceed to a nestin-positive progenitor cell stage. In summary, our NEP platform has generated a framework upon which future experiments can be efficiently designed to dissect the molecular underpinnings of nuclear reprogramming and neuronal cellular maturity. Our novel biological findings uncover stochastic cellular mechanisms that explain to some extent the inefficiencies of induced neuron formation. 


\section{Figure Legends}

Figure 1: 3D NEP Design Delivers High Dose Plasmid DNA at Single Cell Resolution with Lower Biological Variability

(A) Photolithography-based adaptation of Transwell insert into a 3D NEP platform.

(B) SEM of the modified insert surface showing a patterned microwell array (left, scale bar $=50$

$\mu \mathrm{m}$ ) over the nanochanneled membrane. Only exposed nanochannels (right, scale bar $=1 \mu \mathrm{m}$ )

can NEP-transfect the cells loaded on the apical side of the membrane.

(C) Schematic diagram of how the transfection is conducted using the modified Transwell inserts.

(D) Circuit diagram for the in-parallel array of nanochannels illustrating the relationship between the resistance across the membrane and the number of actively nanoporating channels. (E) Schematic illustration of a cell sitting directly on top of a nanoporating channel.

$(\mathbf{F}, \mathbf{G )}$ Finite element modeling of the electric field distribution during nanoporation. The electric field is maximized within the nanochannel which leads to enhanced and highly localized transmembrane potentials on the cells.

(H-J) Bicistronic constructs for Ascl1, Brn2, and Myt1l (color coded green, red, and blue) were introduced into MEFs by NEP. Cells were allowed to recover for 24 hours as indicated on the schematic, and epifluorescent photomicrographs were captured. NEP cells are compared to cells that underwent BEP in parallel experiments. Arrowheads demarcate the same cell in the different panels. Mean fluorescent intensity was obtained from ImageJ and plotted in the respective channels. In addition to showing a higher cellular mean fluorescent intensity, the NEP'ed cells showed a much smaller interquartile range in fluorescent intensities, indicating a more uniform delivery across cells that underwent the NEP. Asterisk indicates statistical significance ( $p<0.05$ by T-test). Box plot shows a solid line at the mean, the height of the box is the interquartile range, and the maximum length of whiskers is 1.5 times the interquartile range. Panels h1 and h2 represent GFP fluorescence as a proxy for Ascl1 transcript expression for BEP, and NEP, and the normalized fluorescence is box-plotted in h3. Panels i1 and i2 represent RFP fluorescence as a proxy for Brn2 transcript expression for BEP, and NEP, and the normalized fluorescence is box-plotted in i3. Panels j1 and j2 represent CFP fluorescence as a proxy for Mytl 1 transcript expression for BEP, and NEP, and the normalized fluorescence is box-plotted in j3.

Figure 2: Persistent long-term expression of BAM reporter constructs in vitro and in vivo.

(A) MEFs were transfected with BAM cDNA expression plasmids designed to have a bicistraonic transcript for fluorescent tagging as follows: Ascl1::GFP, Brn2::RFP, Myt1l::CFP. Cells were differentiated for 21 days. Endogenous fluorescence was captured in an epifluorescence microscope and demonstrates continued expression of the plasmids at 21 days. On day 1 post-NEP, cells were implanted in utero to E15.5 embryos. Pups were analyzed by in vivo imaging on post-natal day 4 and 8 for GFP expression.

(B) Animals were imaged on post-natal day 4 (C-D, each photograph represents a different litter).

Figure 3: Induced Neuron Formation by NEP

(A) TUJ1 quantification of stained cells 7 days post-BEP or post-NEP using either equimolar ratios of plasmid DNA or molar ratios of 1:2:1 of Brn2, Ascl1, and Myt1, respectively. Neuronal reprogramming efficiency (defined as 100Xtotal neuron number/seeding density) is plotted as a function of electroporation condition. Asterisks indicate statistical significance from the three other groups $(p<0.05)$. 
(B-D) Phenotypic characterization of induced neurons post-NEP at 14 days. Molecular markers are indicated on the left of each panel.

(E) Electrophysiology of neurons 20-days post-NEP. Cells display the necessary voltage-gated currents to fire action potentials. Both transient inward currents and sustained outward currents were observed in response to depolarizing voltage simulations.

(F) A typical response to a $20 \mathrm{pA}$ current injection is illustrated and indicates that cells fired action potentials in response to depolarizing current.

(G-H) Voltage-current relationship quantification for the transient and sustained components of whole-cell current. Sustained plateau currents are shown in G, and transient inward currents are shown in $\mathrm{h}$.

(I) Neuronal complexity index of induced neurons by Sholl Analysis. The y-axis is the ratio of mean number of intersections between BAM at 1:2:1/1:1:1, $\mathrm{X}$-axis is distance from neuron cell body. Supplementary Table $\mathrm{S} 4$ shows inferential statistics.

\section{Figure 4: Stochastic Barriers to Neuronal Reprogramming}

(A-B) Time-lapse photography of neuronal reprogramming post-NEP. In these images, the time course is delineated in the box above the figure. White arrow in A-series denotes an Ascl1IRES-GFP-positive cell undergoing degeneration. Red arrow in A-series denotes a cell that first undergoes cell division with one daughter cell undergoing degeneration, with the second cell showing robust reprogramming but then ultimately cellular degeneration. Blue arrow in Bseries shows an Ascl1-IRES-GFP-positive cell without morphological evidence of reprogramming. Yellow arrow in B-series shows an Ascl1-IRES-GFP-positive cell with successful reprogramming that shows robust neurite growth.

(C-C') White arrow denotes cell with silenced Ascl1-GFP but with preserved Brn2-RFP. Clue arrow denotes a cell division resulting in near-symmetric inheritance of Ascl1-GFP but asymetric inheritance of Brn2-RFP. Molecular marker is on left, the time post-NEP is on the top of each panel. Images from cells in panels A and C were started 4-6 hours after NEP of BAM. Cells in panel B were imaged 3 days after NEP.

(D) Endogenous silencing of exogenous Ascl1-IRES-GFP. Note that Ascl1-silenced cells do not become TUJ1 positive.

\section{Figure 5: CCNA2 ablation decreases neuronal reprogramming efficiency}

(A-C) Targeted ablation of CCNA2 early blocks neuronal reprogramming. Experimental schematics are illustrated to the left of each panel. Molecular markers are denoted on the left of each panel. Images represent epifluorescent photomicrographs. Panels a1-a4 represent images obtained from reprogramming of wild-type MEFs with BAM plus cre-GFP 14 days post-NEP. Panels b1-b3 represent images obtained 14 days post-NEP from MEFs obtained from CCNA2fl/fl embryos transfected with $B A M$ plus cre-GFP. Panels c1-c3 represent images obtained from from CCNA2fl/fl MEFs transfected first with BAM, and then infected with adenovirus-cre-GFP 6 hours post-NEP.

(D) $\beta$-tubulin III Qrt-PCR demonstrates significant decrease in $p C r e$ conditions relative to Adeno-cre conditions.

\section{Figure 6: BAM-mediated induced neuron formation by NEP generates nestin- positive cells.}

(A) GFP and nestin co-expression indicates that a subset of Ascl1-ires-GFP expressing cells show nestin immunoreactivity.

(B) Ascl1 and Nestin qRT-PCR demonstrate increased exogenous Ascl1 expression after 10 NEP pulses of BAM and increased endogenous nestin expression after 10 NEP pulses of BAM 
(asterisk indicates $\mathrm{p}<0.05$ ). Schematic shows general methodology to test for nestin progenitor cell requirement for BAM-mediated neuronal reprogramming.

(C) Quantification of GFP positive cells after NEP of either phosphate buffered saline (PBS) or BAM. Note that by day 14, a significant population of GFP-positive cells is noted indicating increased cre-mediated recombination.

(D-F) Epifluorescent photomicrographs at different stages of the reprogramming of MEFs derived from Nestin-cre-ER, Rosa ${ }^{m T d T o m a t o / m G F P}$ mice treated with BAM (1:2:1 ratio) plust 4OHT. Under these conditions, cre recombinase would be active in cells that express nestin. Panels d1- $\mathrm{d} 3$ are 1 day post plating. Panels e1-e 3 are 3 days post-plating. Panels f1-f3 are 14 days post-plating. Molecular markers are delineated on the boxes to the left of the panel. 


\section{Author Contributions}

- NEP design, manufacturing, and validation was performed by Daniel Gallego-Perez, Veysi Malkoc, Junyu Ma, Wei-Ching Liao, Xi Zhao, Xinmei Wang, Andrew Adler, Kam Leong and L. James Lee.

- Design, implementation, and interpretation of reprogramming experiments, live cell imaging, transgenic mouse experiments, histology, photomicroscopy, and neuronal morphometric studies were performed by Daniel Gallego-Perez, José J. Otero, Catherine Czeisler, Patrick Gygli, Fay Patsy Catacutan, Cristina Ortiz, Hamza Gokozan, Veysi Malkoc, Subhadip Ghatak, and Aaron Cowgill.

- In utero surgery experiments were developed, implemented, and validated by Brian Wulff, Subhadip Ghatak, Surya Gnyawali, Daniel Gallego-Perez, Traci A. Wilgus, Savita Khanna, Cameron Rink, and Chandan K. Sen

- Electrophysiology was performed and interpreted by Thomas Sherwood and Candice Askwith.

- The manuscript was written by José Javier Otero, Daniel Gallego-Perez, L. James Lee, and Chandan K. Sen with significant feedback and proofreading from all authors.

\section{COMPETING FINANCIAL INTERESTS}

The authors declare no competing financial interests. 


\section{References}

1. K. Takahashi and S. Yamanaka, Induction of pluripotent stem cells from mouse embryonic and adult fibroblast cultures by defined factors. Cell. 2006;126:663-76 T. Vierbuchen, A. Ostermeier, Z. P. Pang, Y. Kokubu, T. C. Sudhof and M. Wernig, Direct conversion of fibroblasts to functional neurons by defined factors. Nature. 2010;463:103541

Z. Li and T. M. Rana, A kinase inhibitor screen identifies small-molecule enhancers of reprogramming and iPS cell generation. Nature communications. 2012;3:1085

K. Li, S. Zhu, H. A. Russ, S. Xu, T. Xu, Y. Zhang, et al., Small molecules facilitate the reprogramming of mouse fibroblasts into pancreatic lineages. Cell Stem Cell. 2014;14:22836

H. Wang, N. Cao, C. I. Spencer, B. Nie, T. Ma, T. Xu, et al., Small Molecules Enable Cardiac Reprogramming of Mouse Fibroblasts with a Single Factor, Oct4. Cell reports. 2014 M. L. Liu, T. Zang, Y. Zou, J. C. Chang, J. R. Gibson, K. M. Huber, et al., Small molecules enable neurogenin 2 to efficiently convert human fibroblasts into cholinergic neurons. Nature communications. 2013;4:2183

J. Ladewig, J. Mertens, J. Kesavan, J. Doerr, D. Poppe, F. Glaue, et al., Small molecules enable highly efficient neuronal conversion of human fibroblasts. Nat Methods.

2012;9:575-8

T. Geng and C. Lu, Microfluidic electroporation for cellular analysis and delivery. Lab on a chip. 2013;13:3803-21

P. E. Boukany, A. Morss, W. C. Liao, B. Henslee, H. Jung, X. Zhang, et al., Nanochannel electroporation delivers precise amounts of biomolecules into living cells. Nature nanotechnology. 2011;6:747-54

C. Sun, Z. Cao, M. Wu and C. Lu, Intracellular tracking of single native molecules with electroporation-delivered quantum dots. Analytical chemistry. 2014;86:11403-9

S. Ma, B. Schroeder, C. Sun, D. N. Loufakis, Z. Cao, N. Sriranganathan, et al., Electroporation-based delivery of cell-penetrating peptide conjugates of peptide nucleic acids for antisense inhibition of intracellular bacteria. Integrative biology : quantitative biosciences from nano to macro. 2014;6:973-8

K. Okita, H. Hong, K. Takahashi and S. Yamanaka, Generation of mouse-induced pluripotent stem cells with plasmid vectors. Nature protocols. 2010;5:418-28 A. F. Adler, C. L. Grigsby, K. Kulangara, H. Wang, R. Yasuda and K. W. Leong, Nonviral direct conversion of primary mouse embryonic fibroblasts to neuronal cells. Molecular therapy. Nucleic acids. 2012;1:e32

X. Chen, E. Pham and K. Truong, TEV protease-facilitated stoichiometric delivery of multiple genes using a single expression vector. Protein science : a publication of the Protein Society. 2010;19:2379-88

H. Hirai, T. Tani, N. Katoku-Kikyo, S. Kellner, P. Karian, M. Firpo, et al., Radical acceleration of nuclear reprogramming by chromatin remodeling with the transactivation domain of MyoD. Stem Cells. 2011;29:1349-61

D. Levanon, O. Brenner, V. Negreanu, D. Bettoun, E. Woolf, R. Eilam, et al., Spatial and temporal expression pattern of Runx3 (Aml2) and Runx1 (Aml1) indicates non-redundant functions during mouse embryogenesis. Mech Dev. 2001;109:413-7

J. F. Brunet and A. Pattyn, Phox2 genes - from patterning to connectivity. Curr Opin Genet Dev. 2002;12:435-40

O. L. Wapinski, T. Vierbuchen, K. Qu, Q. Y. Lee, S. Chanda, D. R. Fuentes, et al., Hierarchical mechanisms for direct reprogramming of fibroblasts to neurons. Cell. 2013;155:621-35 
Y. Katsuno, A. Suzuki, K. Sugimura, K. Okumura, D. H. Zineldeen, M. Shimada, et al., Cyclin A-Cdk1 regulates the origin firing program in mammalian cells. Proceedings of the National Academy of Sciences of the United States of America. 2009;106:3184-9

J. Kim, S. C. Su, H. Wang, A. W. Cheng, J. P. Cassady, M. A. Lodato, et al., Functional integration of dopaminergic neurons directly converted from mouse fibroblasts. Cell Stem Cell. 2011;9:413-9

J. A. Bernal, RNA-based tools for nuclear reprogramming and lineage-conversion: towards clinical applications. Journal of cardiovascular translational research. 2013;6:956-68 X. Guo, Q. Liu, G. Wang, S. Zhu, L. Gao, W. Hong, et al., microRNA-29b is a novel mediator of Sox2 function in the regulation of somatic cell reprogramming. Cell Res. 2013;23:14256

L. Warren, P. D. Manos, T. Ahfeldt, Y. H. Loh, H. Li, F. Lau, et al., Highly efficient reprogramming to pluripotency and directed differentiation of human cells with synthetic modified mRNA. Cell Stem Cell. 2010;7:618-30

P. Perez-Pinera, D. D. Kocak, C. M. Vockley, A. F. Adler, A. M. Kabadi, L. R. Polstein, et al., RNA-guided gene activation by CRISPR-Cas9-based transcription factors. Nat Methods. 2013;10:973-6

J. Hanna, K. Saha, B. Pando, J. van Zon, C. J. Lengner, M. P. Creyghton, et al., Direct cell reprogramming is a stochastic process amenable to acceleration. Nature. 2009;462:595601

Y. Rais, A. Zviran, S. Geula, O. Gafni, E. Chomsky, S. Viukov, et al., Deterministic direct reprogramming of somatic cells to pluripotency. Nature. 2013;502:65-70

Y. Nakada, T. L. Hunsaker, R. M. Henke and J. E. Johnson, Distinct domains within Mash1 and Math1 are required for function in neuronal differentiation versus neuronal cell-type specification. Development. 2004;131:1319-30

D. S. Castro, B. Martynoga, C. Parras, V. Ramesh, E. Pacary, C. Johnston, et al., A novel function of the proneural factor Ascl1 in progenitor proliferation identified by genomewide characterization of its targets. Genes Dev. 2011;25:930-45

B. Li, T. Su, R. Ferrari, J. Y. Li and S. K. Kurdistani, A unique epigenetic signature is associated with active DNA replication loci in human embryonic stem cells. Epigenetics. 2014;9:257-67 
3D NEP DESIGN

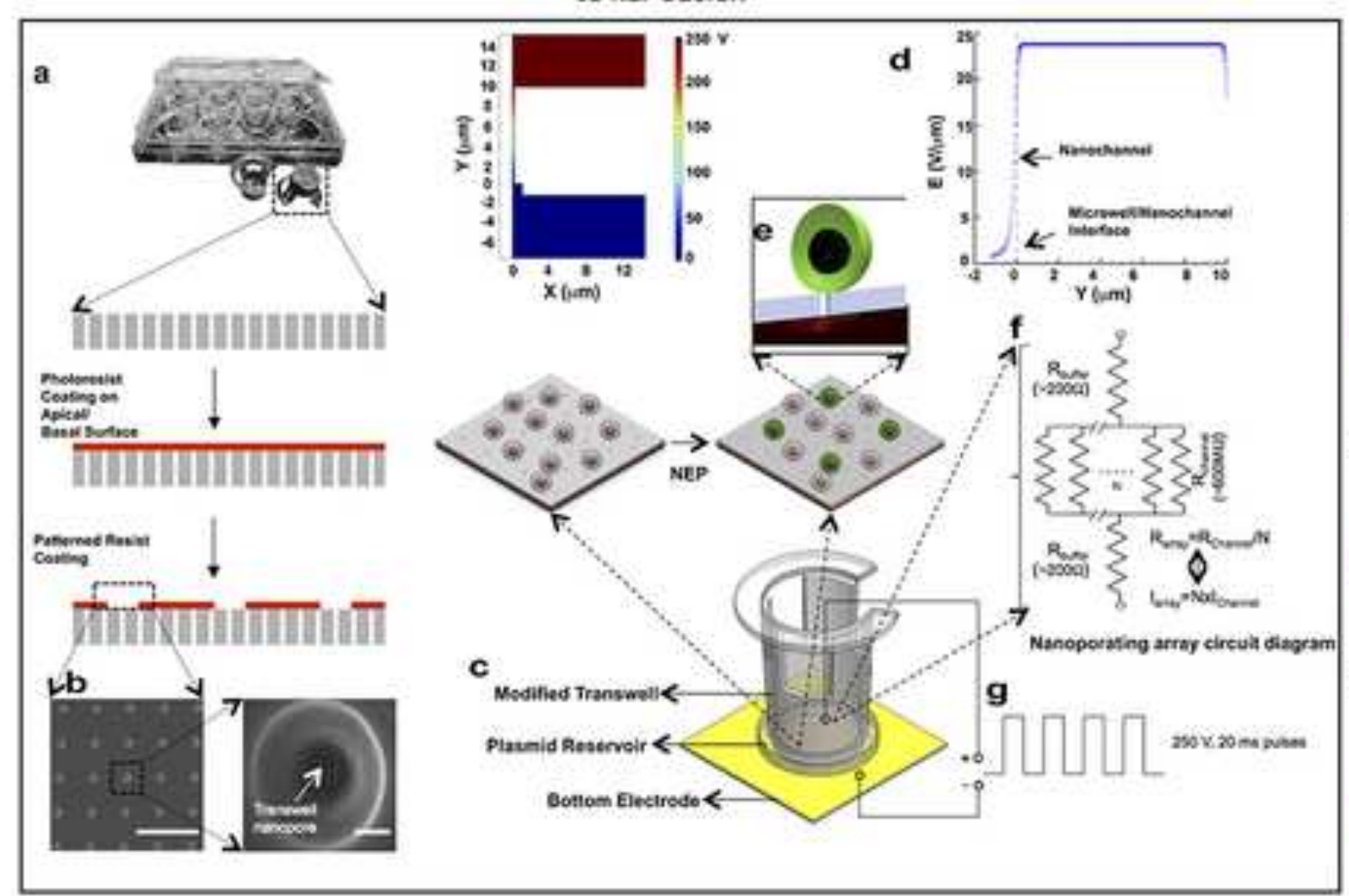

NEP (5 Pulses) OR BEP

DAYO

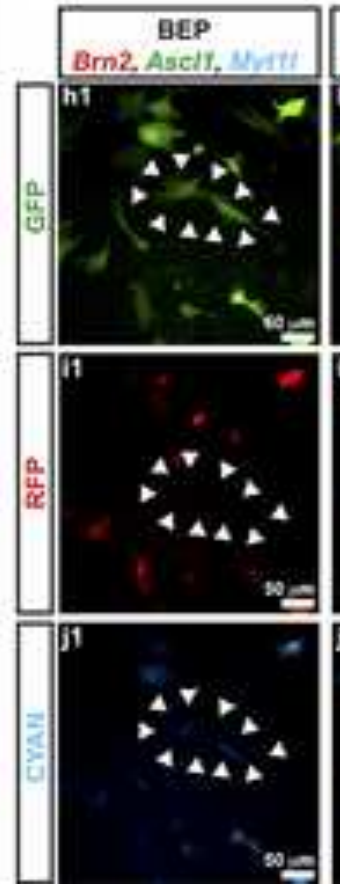

Gallego-Perez, Ocora, ot aL Figure 1.

IMAGE CAPTURE 24 hr

i

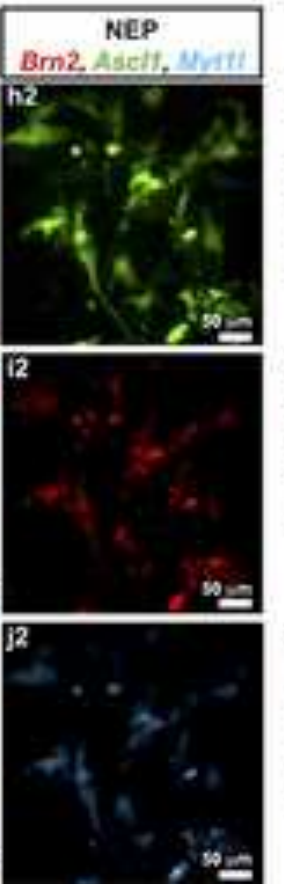

Relative Fluorescence

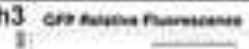
$z_{1}^{1}$ * $\mid \begin{aligned} & 1 \\ & 1\end{aligned}$

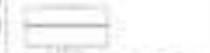

stelloomen corions

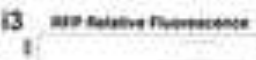
引. $\star$ is $\sqrt{t}$

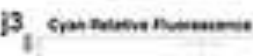
站 $\int_{i}^{2}$

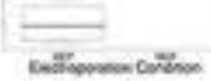




\section{LONG-TERM GENE EXPRESSION IN VITRO}

NEP (5 Pulses), Brn2, Ascl1, Myt11 DAY 0

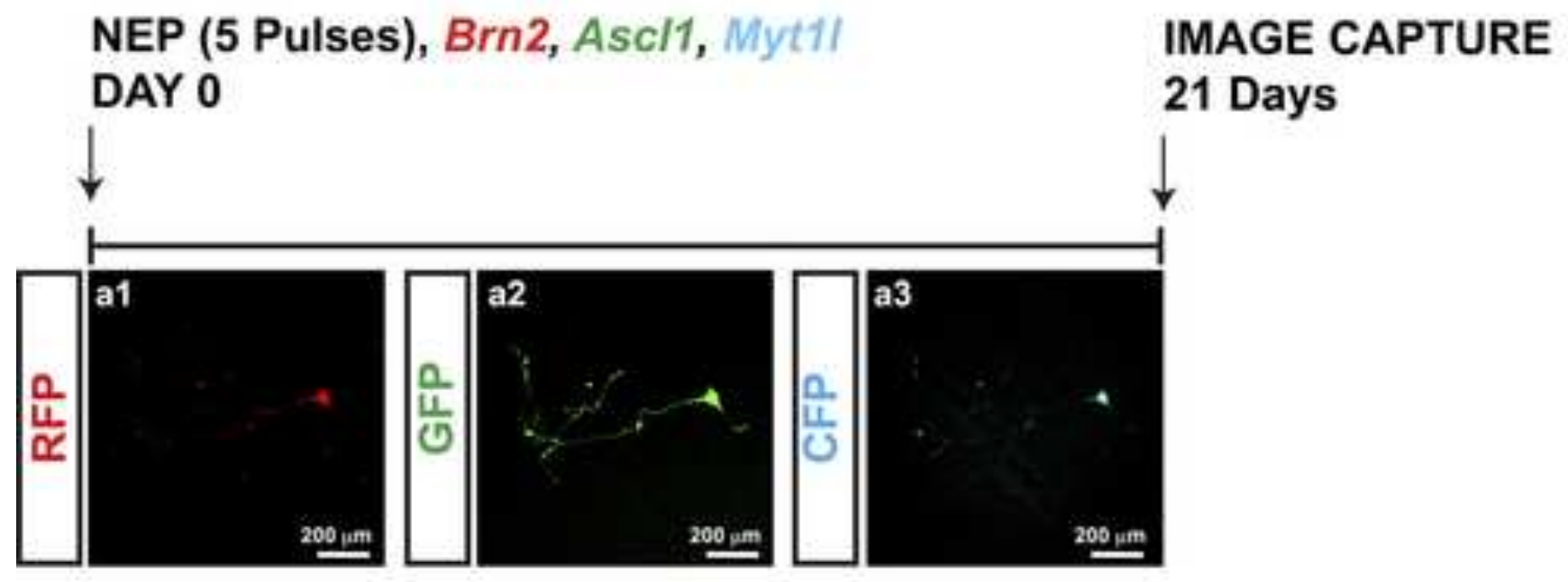

IMAGE CAPTURE 21 Days
P4 IMAGES

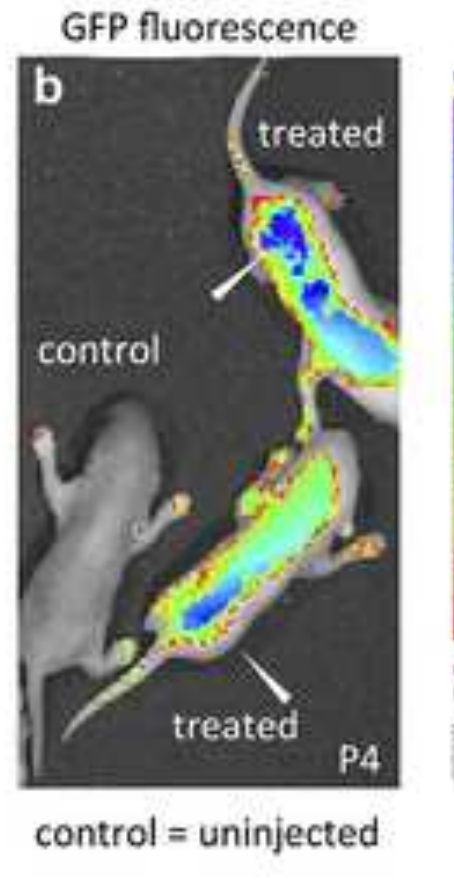

control $=$ uninjected

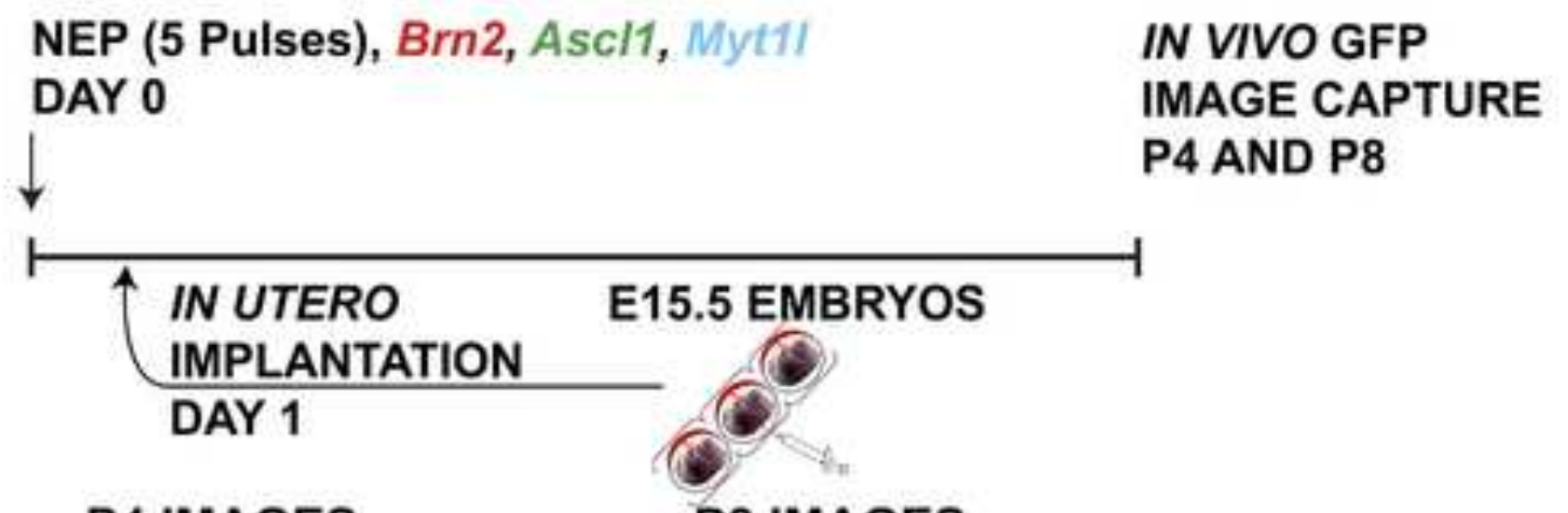

a3

4

$200 \mu \mathrm{m}$
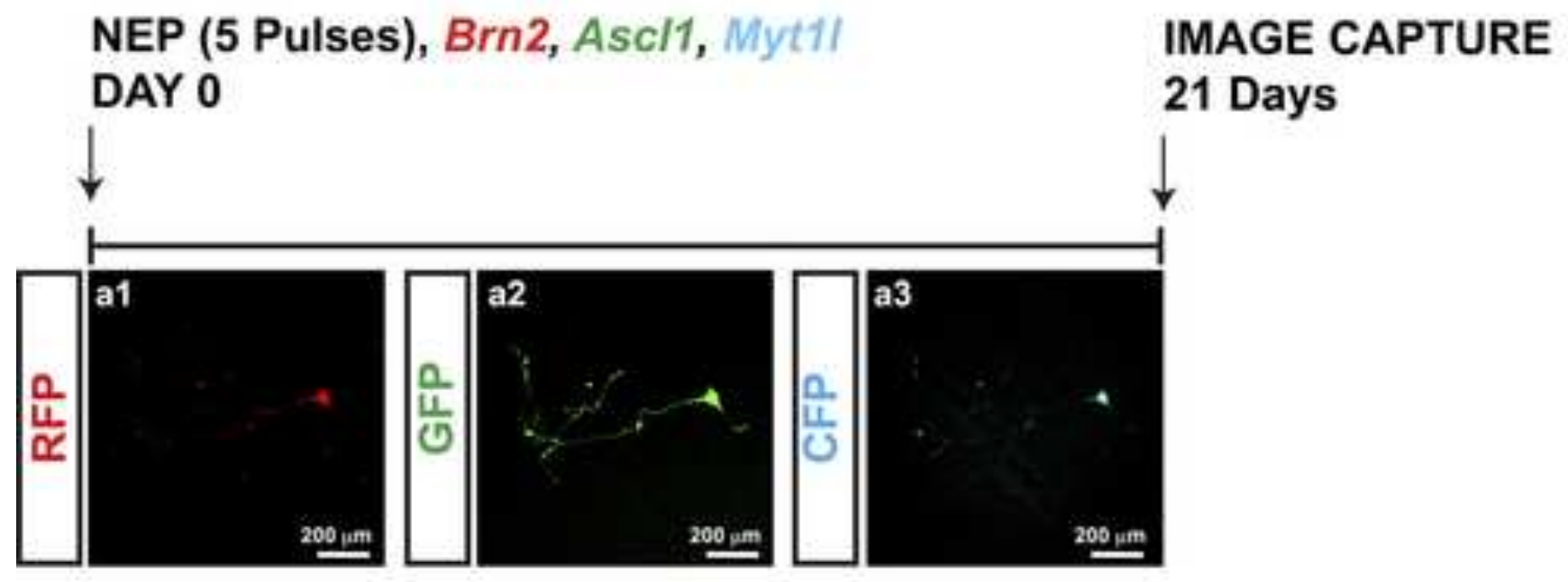

Gallego-Perez, Otero, et al. Figure 2

\section{E15.5 EMBRYOS}

IN VIVO GFP
IMAGE CAPTURE
P4 AND P8

IN VIVO GFP
IMAGE CAPTURE
P4 AND P8

IN VIVO GFP
IMAGE CAPTURE
P4 AND P8

\section{P8 IMAGES}
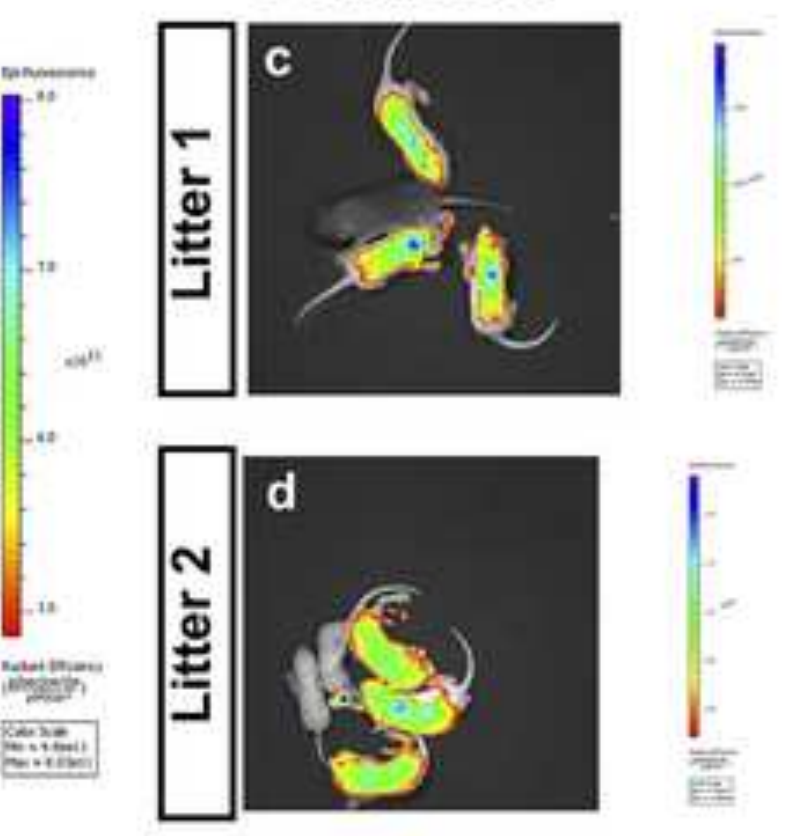
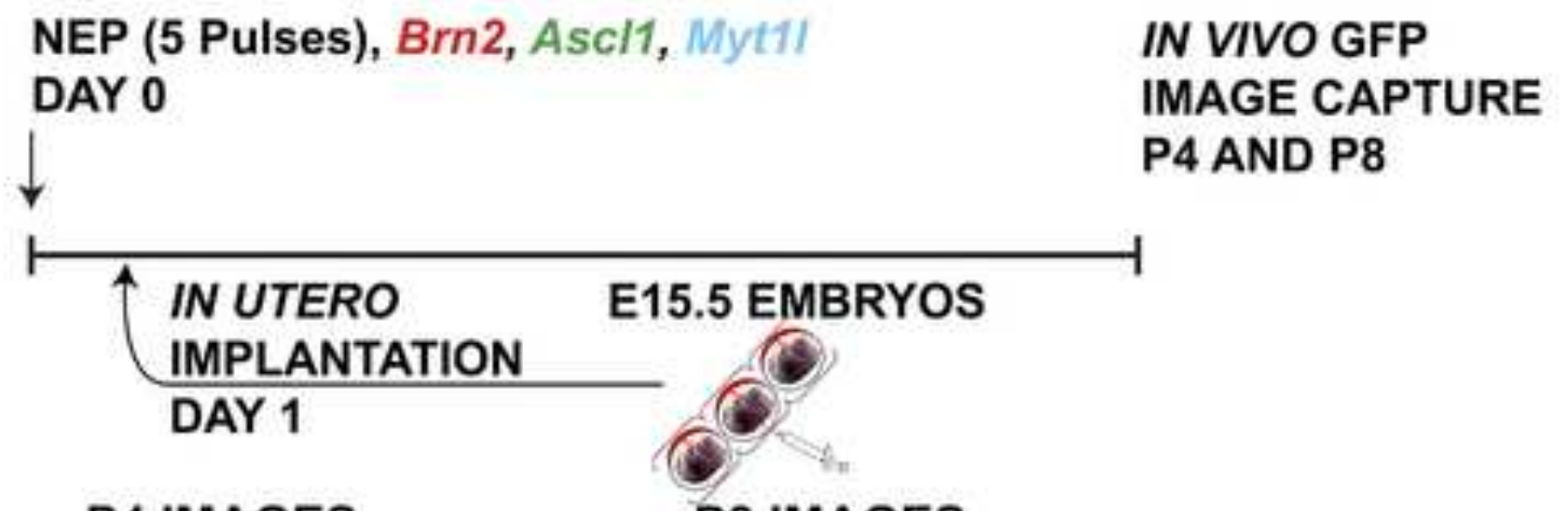

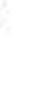


Gallego-Perez, Otero, et al. Figure 3

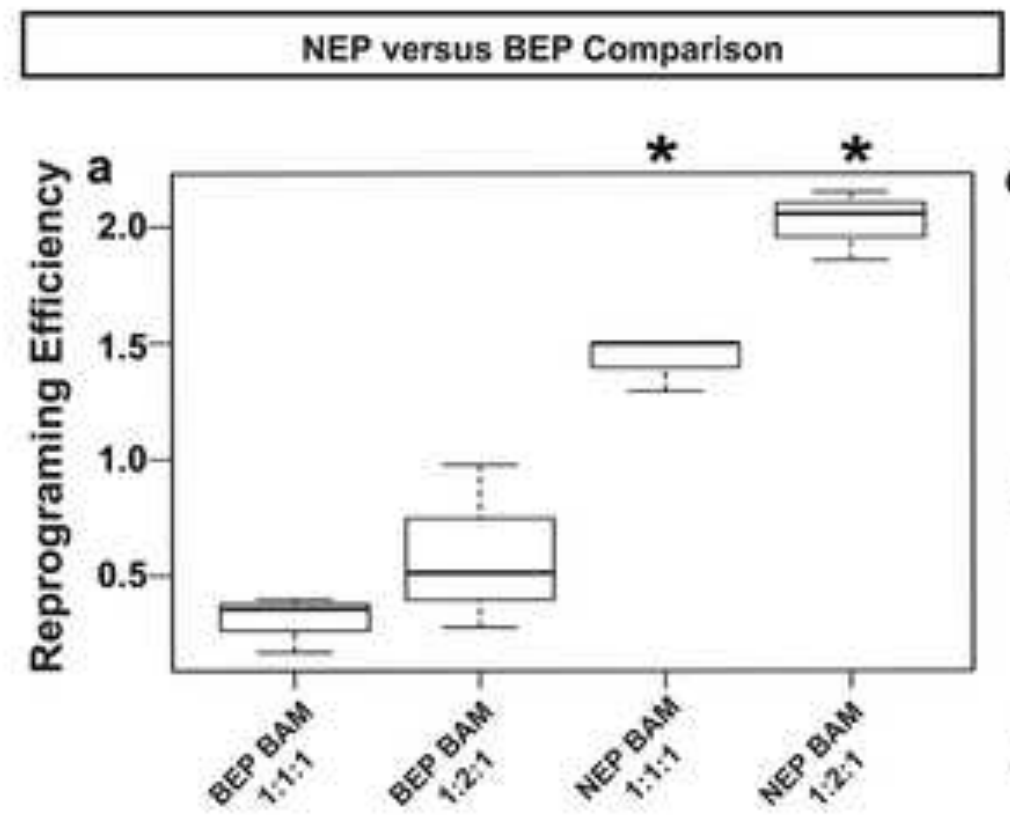

Electrophysiological Analysis of iN's from NEP
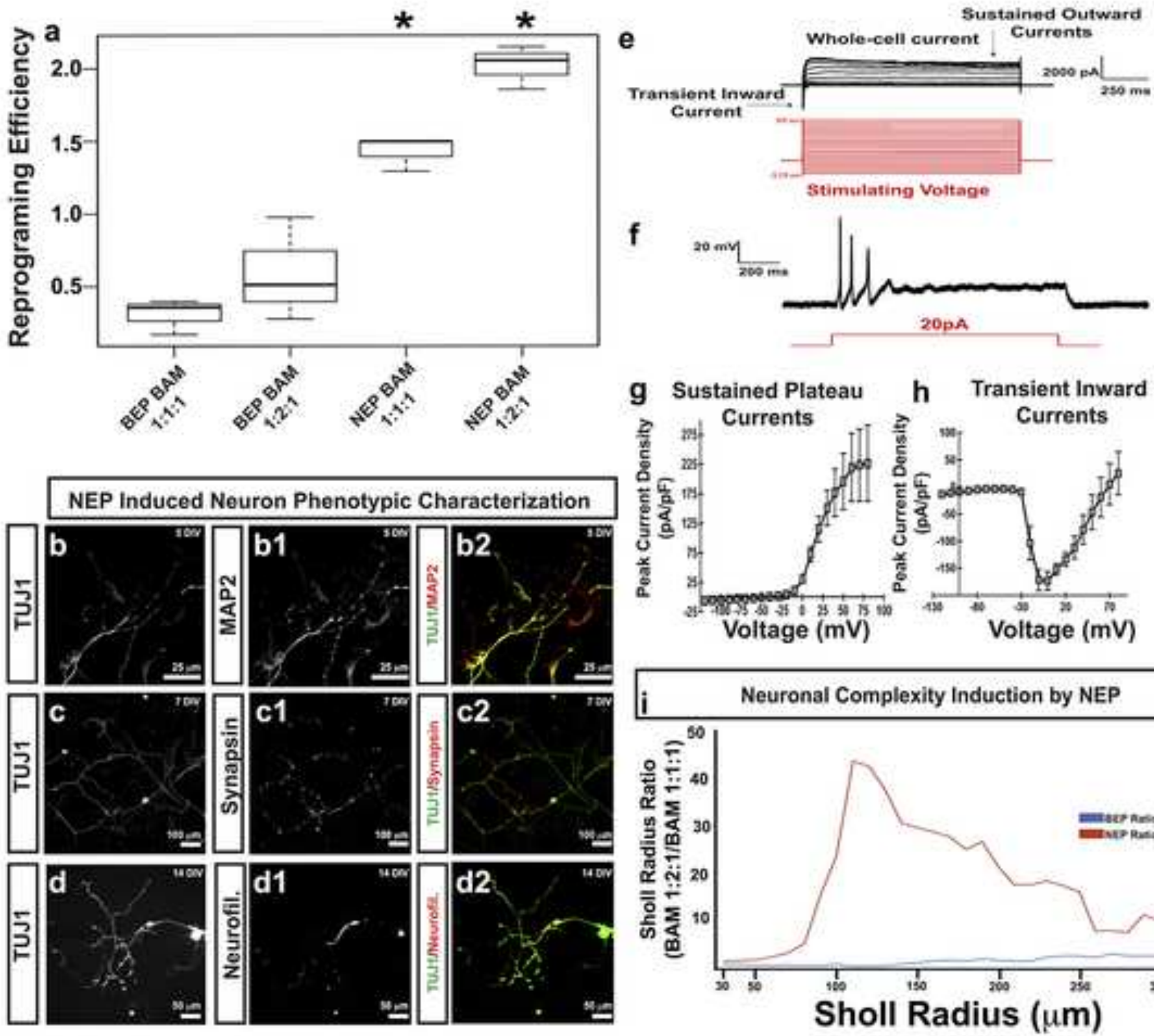

g Sustained Plateau h Transient Inward
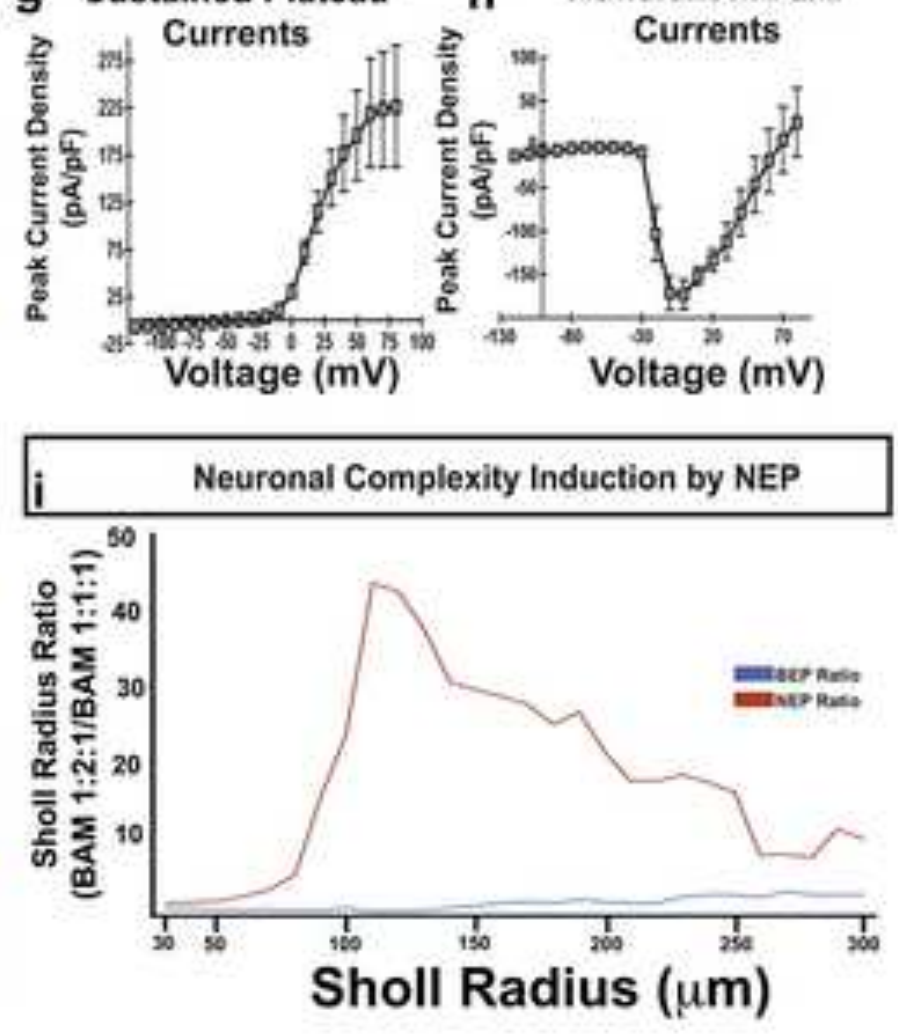
Gallego.Perez, Otero, et al., Figure 4

Live Image Analysis Demostrating Different Reprograming Kinetics post-NEP of BAM (1:2:1)
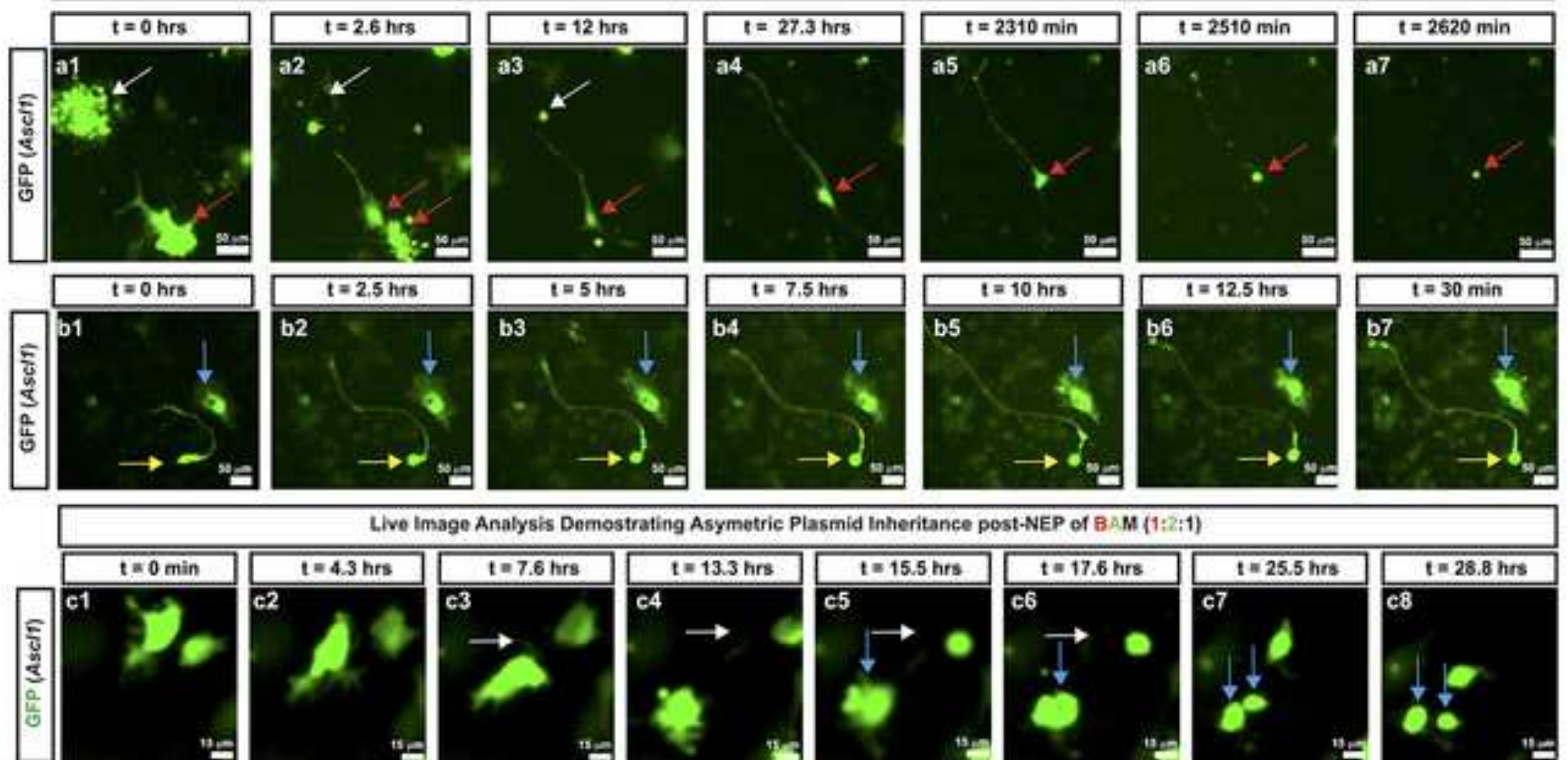

Live Image Analysis Demostrating Asymetric Plasmid Inheritance post-NEP of B.M $(1: 2 ; 1)$
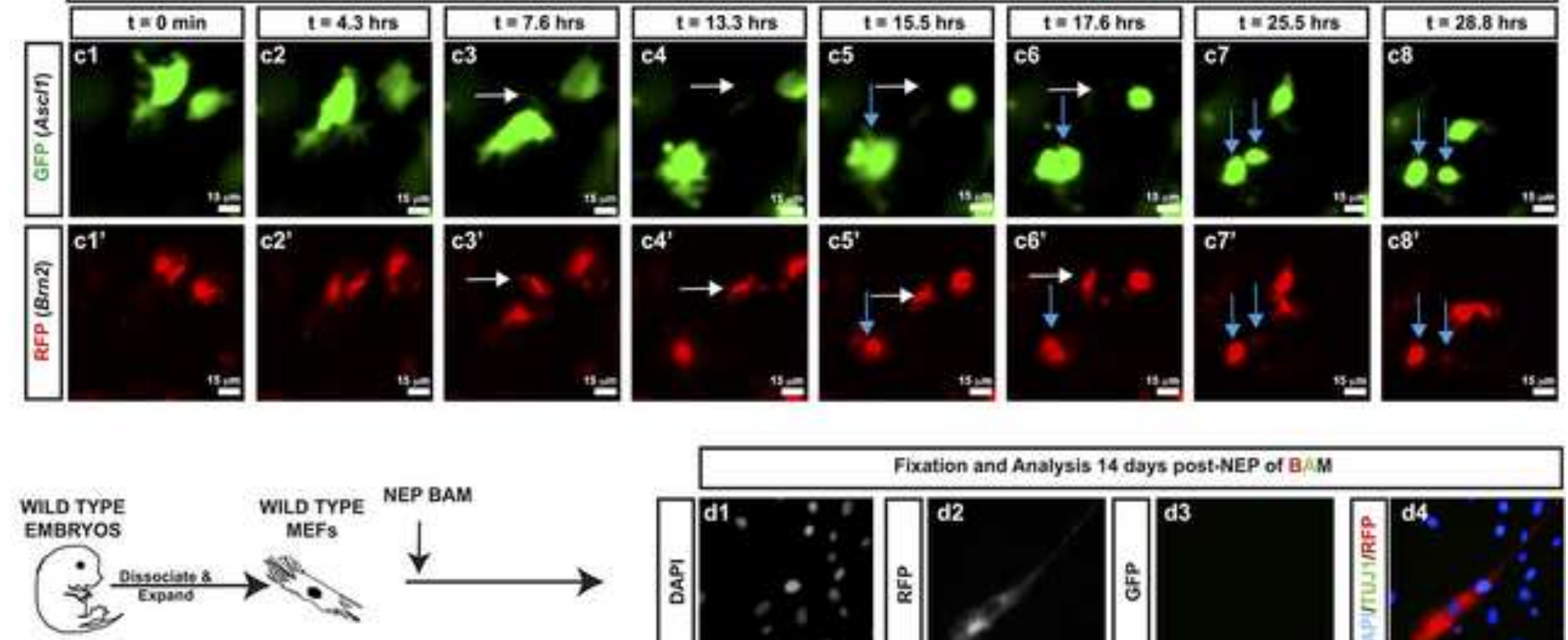

NEP BAM
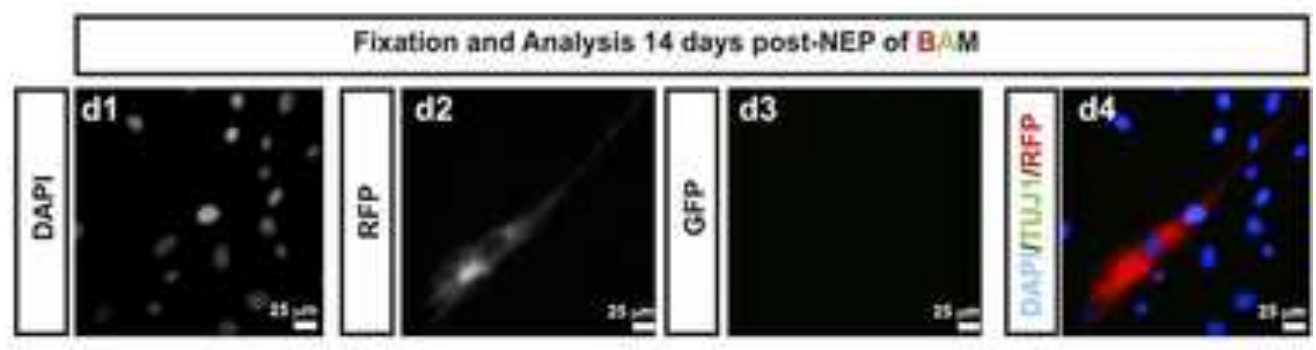
Gallego.Perez, Otero, et al., Figure 5

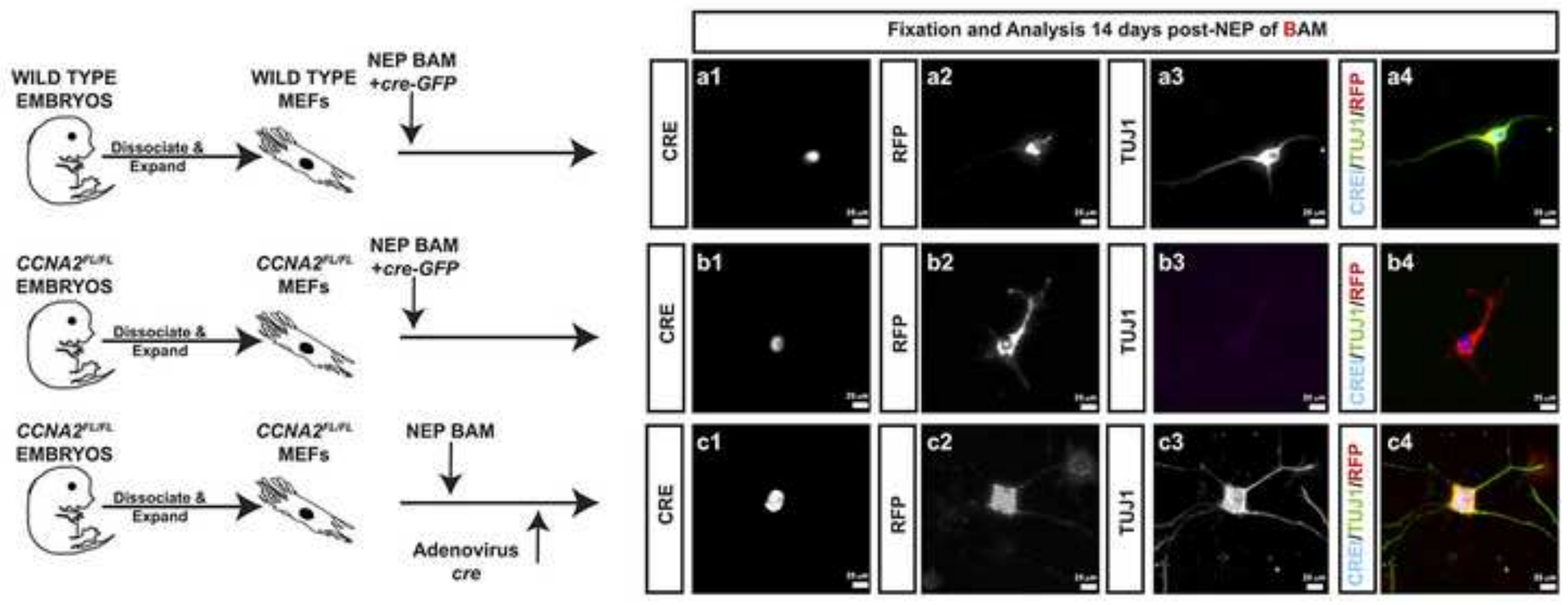

d
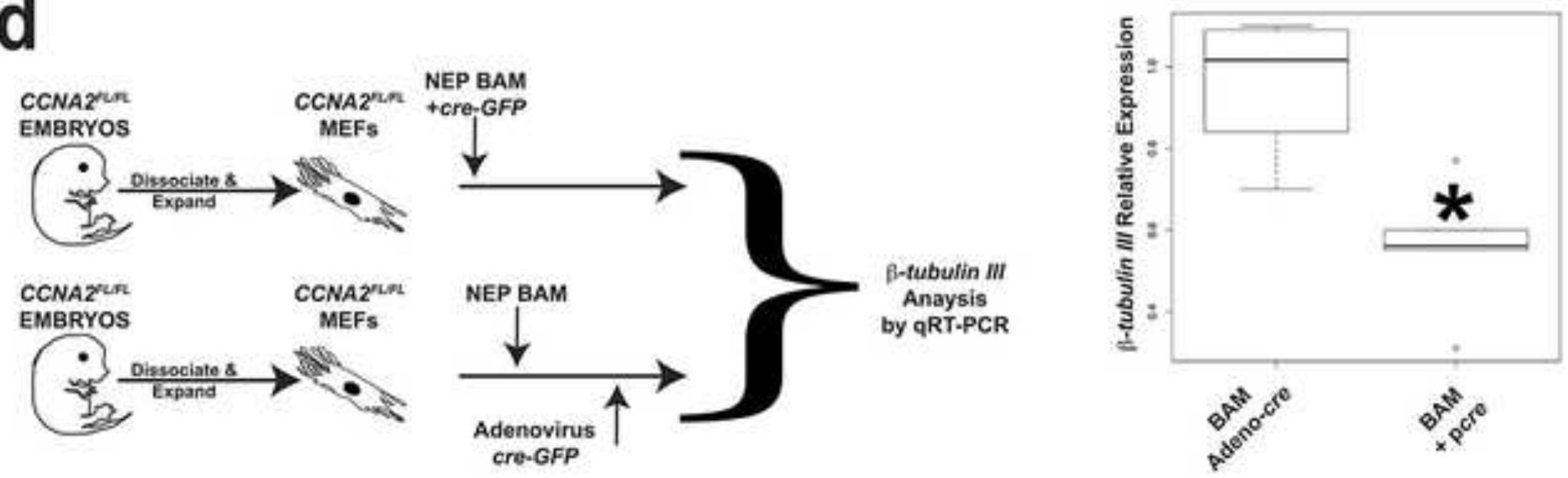
Gallego-Perez, Otero, et al., Figure 6
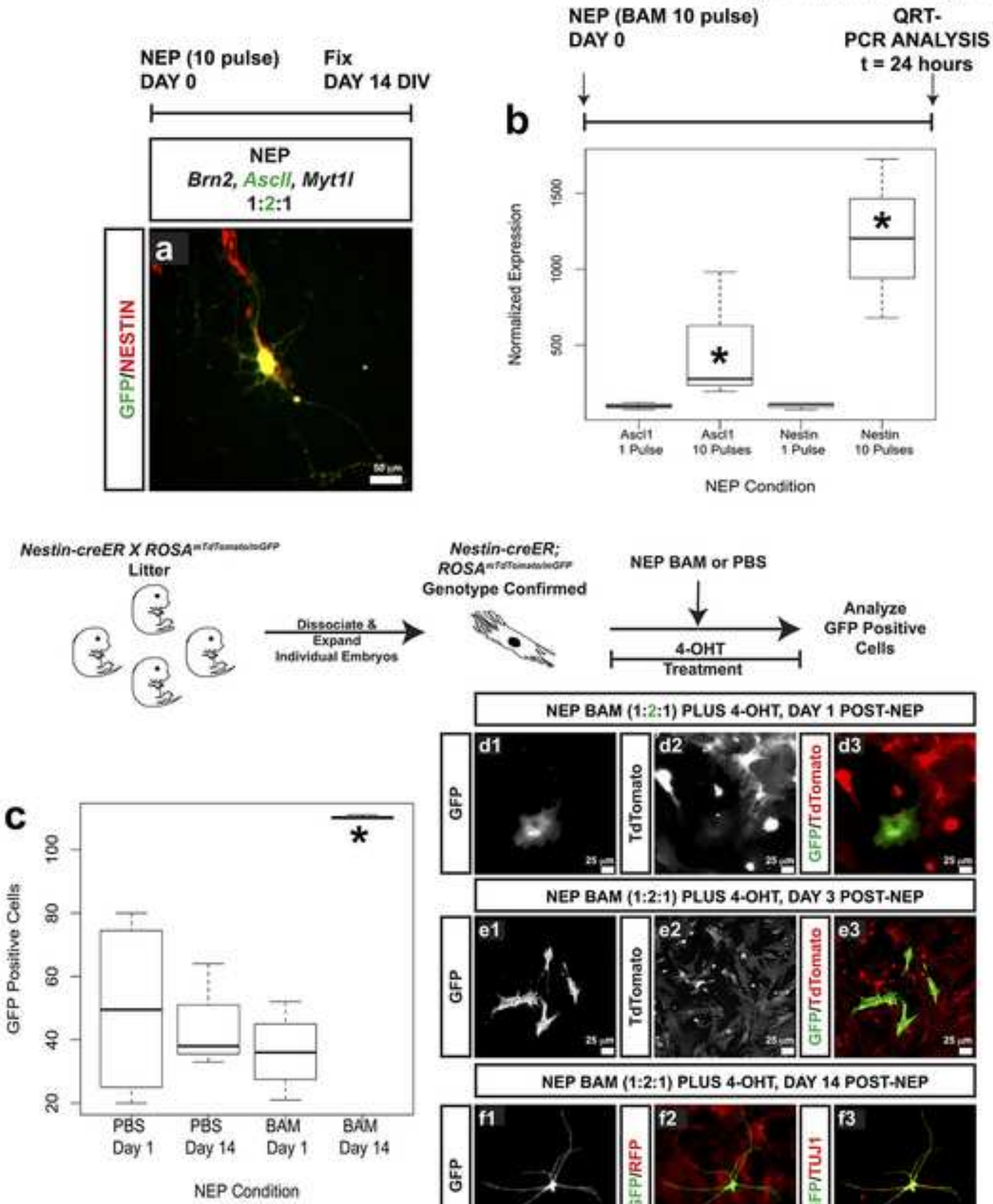

Nostin-croER;

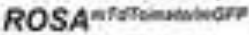

Genotype Confirmed
NEP BAM or PBS

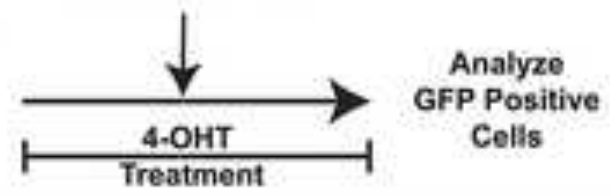

NEP BAM (1:2:1) PLUS 4-OHT, DAY 1 POST-NEP

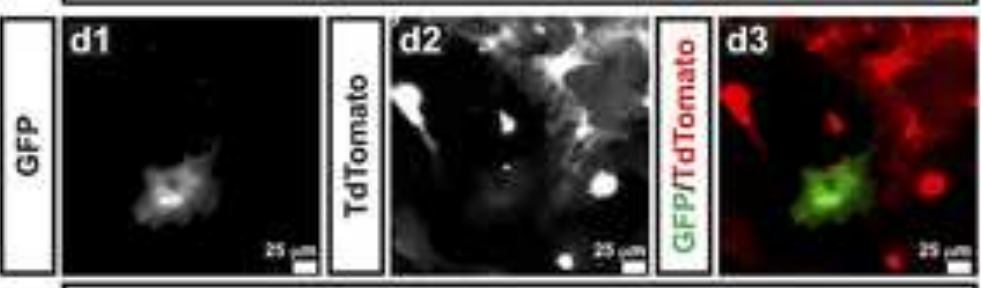

NEP BAM (1:2:1) PLUS 4-OHT, DAY 3 POST-NEP

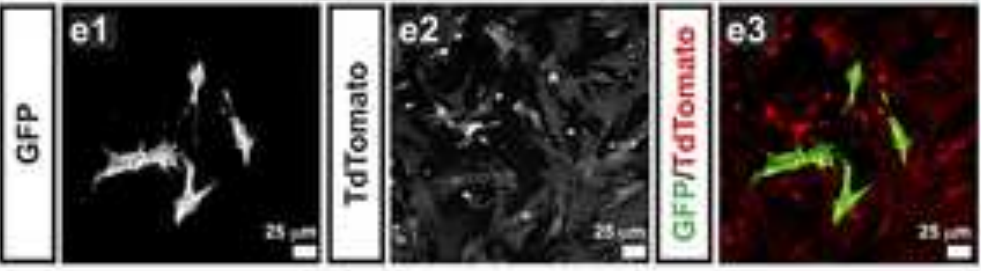

NEP BAM (1:2:1) PLUS 4-OHT, DAY 14 POST-NEP

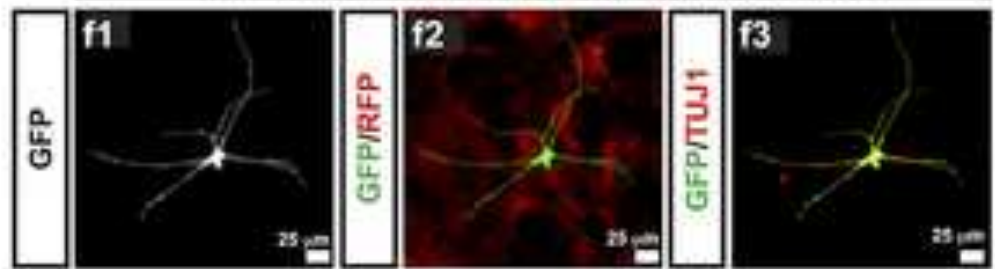




\section{Deterministic Transfection Drives Efficient Nonviral Reprogramming and Uncovers Reprogramming Barriers}

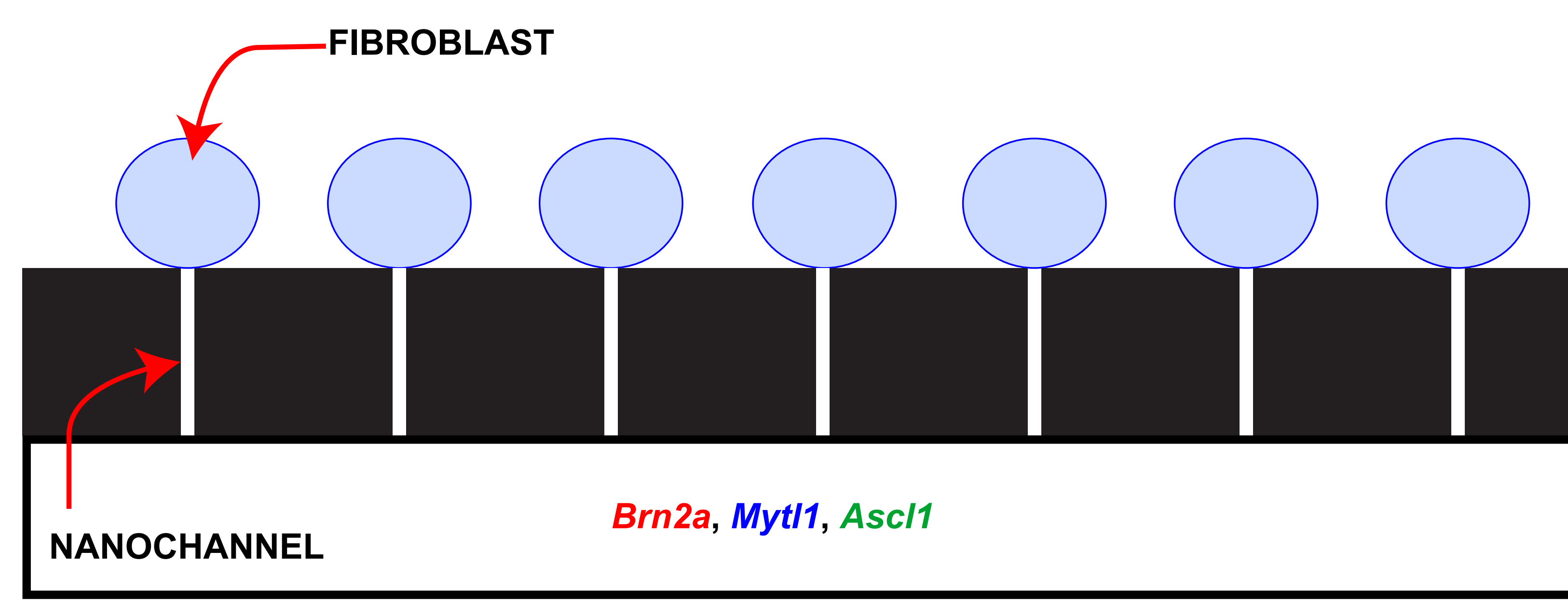

DETERMINISTIC PLASMID DELIVERY BY NOVEL VERTICAL NANOCHANNEL ELECTROPORATION

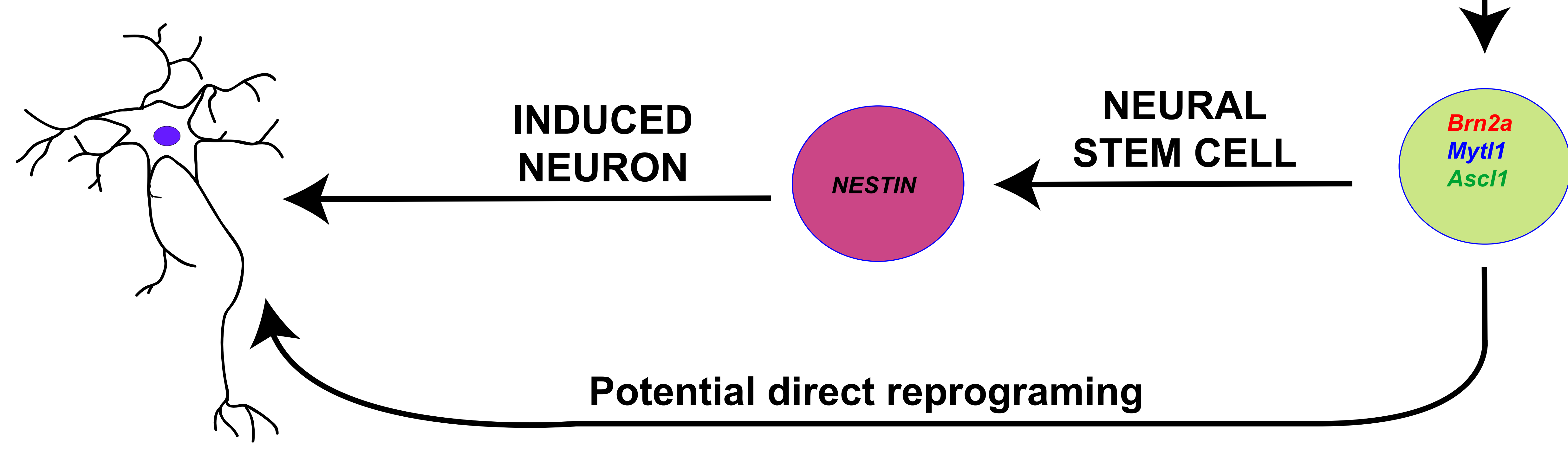

